\title{
Impact of Supply Chain Coordination for Deteriorating Goods with Stock-Dependent Demand Rate
}

\author{
Chuanxu Wang \\ School of Economy and Management, Shanghai Maritime University Shanghai, China \\ Email: cxwang@shmtu.edu.cn
}

\begin{abstract}
To analyze effects of supply chain coordination for deteriorating goods with stock-dependent demand rate, this paper presents decision models for order quantity and ordering cycle under two scenarios( decentralized supply chain, centralized supply chain). Numerical study is carried out to demonstrate the effectiveness of the proposed models, and to analyze the impact of supply chain coordination on supply chain profit. Sensitivity analysis is performed to study the impact of different parameters associated with the model, such as the rate of deterioration, the retailer's purchase cost, the manufacturer's production cost, the retailer's and manufacturer's holding cost on the supply chain profit increase percentages generated by the supply chain coordination.
\end{abstract}

Keywords: supply chain coordination, deteriorating goods, ordering cycle, stock-dependent demand

\section{Introduction}

In real life, many inventory goods, such as agricultural products, fashion goods, drugs and high-tech products, are subject to depletion through spoilage, shrinkage, decay and obsolescene [1]. The deterioration is quite prevalent and should not be disregarded. Inventory management for deterioration goods has received many attentions from researchers and practitioners. Most of the existing researches focus on the EOQ-based inventory decision models. Ghare and Schrader [2] presented the EOQ model by considering the combined effects of demand, usage and linear decay. Covert and Philip [3]used the variable deterioration rate of the two-parameter Weibull distribution, to formulate the inventory decision model under the assumptions of a constant demand rate, with no shortages allowed. Philip [4] modified this model by using the deterioration rate of the three-parameter Weibull distribution. Tadikamalla [5] adopted gamma distributed deterioration under constant demand over time, without shortages. Moon and Lee [6] presented the EOQ model with a normally distributed deterioration rate. Other deterioration inventory models have extended prior research by considering a time-varying demand function, with or without shortages. Dave and Patel [7] proposed an EOQ model under time-proportional demand, with no shortages allowed. Sachan [8] extended their model by considering shortages. Bahari-Kashani [9] generalized the problem by permitting variations in both replenishment cycle length and order quantity. Bose et al [10]developed an EOQ model for deterioration items incorporating the effects of inflation, time value of money, a linearly time-dependent demand rate and shortages. Replenishment decision models under time-proportional demand and exponentially decaying deterioration rate was developed in [1].

It is observed that large quantities of consumer goods displayed in a supermarket generate higher demands. Silver and Peterson [11] noted that the sales at the retail level tend to be proportional to the inventory displayed. Gupta and Vrat [12], Mandal and Phaujdar [13], Baker and Urban [14], Datta and Pal [15], etc developed the EOQ models with stock-dependent demand rate. Mandal and Phaujdar [16], Pal et al. [17] developed the inventory models for deteriorating items with stock-dependent demand rate. In this paper, we have extended these works, on deteriorating inventory research, by considering deteriorating goods with stock-dependent demand in a two-echelon supply chain consisting one manufacturer and one retailer, the objective is to investigate the effects of supply chain coordination on profit increase in the supply chain, and study the impact of different parameters associated with the model, such as the rate of deterioration, the retailer's purchase cost, the manufacturer's production cost, the retailer's and manufacturer's holding cost on the supply chain profit increase percentages generated by the supply chain coordination.

\section{Assumptions and Notations}

\subsection{Assumptions}

(1) The retailer replenishes the stocks from the exclusive source on an EOQ basis. Replenishments are instantaneous.

(2) Lead time is assumed to be zero for the sake of simplicity. 
(3) No backorders are allowed.

(4) Demand rate is dependent on the instantaneous inventory level. The demand rate $d(I)$ of the item, when the inventory is $I$, is considered in the form $d(I)=\alpha I^{\beta}$, where $\alpha>0$ and $0<\beta<1$ are scale and shape parameters ( Baker and Urban 1988).

(5) The manufacturer's production rate is greater than or equal to the demand rate facing the retailer.

(6)The manufacturer is a make-to-order producer; it has a lot-for-lot production policy in response to the retailer's demand. In this particular case, the length of the manufacturer's production cycle is equal to the length of the retailer's replenishment cycle.

\subsection{Notations}

$p$ the sale price for the retailer;

$S$ the order cost per order for the retailer;

$M$ the setup cost per lot for the manufacturer;

$c^{r}\left(c^{m}\right)$ procurement (manufacturing ) cost per unit for retailer (manufacturer);

$d$ the demand rate of the item in the marketplace;

$q$ the production rate;

$h^{r}\left(h^{m}\right)$ the inventory holding cost as a fraction of the inventory cost for the retailer(manufacturer);

$\theta(t)$ the deterioration rate facing both the retailer and manufacturer, $0 \leq \theta \leq 1$;

$I^{r}(t), I^{m}(t)$ inventory level at time $t$ for the retailer ( manufacturer);

$Q$ the order quantity for the retailer;

$T$ the replenishment cycle (or production cycle ) for the retailer( manufacturer);

\section{Basic Model}

In this section, we first derive the profit model for the decentralized supply chain. Later, we present the profit model derived by considering the centralized supply chain.

\subsection{The Decentralized Supply Chain}

In the decentralized supply chain, each entity within the supply chain aims to maximize its own profit functions, with no consideration given to its counterpart's reaction or profit. The retailer makes a replenishment decision based on an EOQ policy that includes inventory holding cost and ordering cost.

During the replenishment cycle, the change in retailer's inventory level depends on demand and deterioration and is given by [1]:

$$
\frac{d I^{r}(t)}{d t}+\theta I^{r}(t)=-\alpha\left[I^{r}(t)\right]^{\beta} \quad 0 \leq t \leq T,
$$

As shown in Pal et al. (1993) [17], equation (1) can be rewritten as

$$
\frac{1}{\theta}\left\{\frac{1}{I^{r}(t)}-\frac{\alpha\left[I^{r}(t)\right]^{\beta-2}}{\theta+\alpha\left[I^{r}(t)\right]^{\beta-1}}\right\} d I^{r}(t)=-d t
$$

By integrating, we get

$$
\ln \frac{\left[I^{r}(t)\right]^{\beta-1}}{\theta+\alpha\left[I^{r}(t)\right]^{\beta-1}}=\ln e^{(1-\beta) \vartheta t}+\ln C_{1},
$$

where $C_{1}$ is integration constant.

It can be rearranged as

$$
\frac{1}{\alpha+\theta\left[I^{r}(t)\right]^{1-\beta}}=C_{1} e^{(1-\beta) \theta t}
$$

By using the boundary condition on inventory $I^{r}(0)=Q$, we can get

$$
C_{1}=\frac{1}{\alpha+\theta Q^{1-\beta}}
$$

Substituting (5) in (4), the retailer's inventory level at time $t(0 \leq t \leq T)$ can be expressed as

$$
I^{r}(t)=\left[\left(\alpha+\theta Q^{1-\beta}\right) e^{-(1-\beta) \theta t}-\alpha\right]^{1 /(1-\beta)} / \theta^{1 /(1-\beta)}
$$

The inventory holding cost in a cycle for the retailer is

$$
H C^{r}=h^{r} c^{r} \int_{0}^{T} I^{r}(t) d t
$$

The retailer's total number of deteriorated goods in a cycle is given by

$$
\begin{gathered}
Q^{D, r}=Q-I^{r}(T)-\int_{0}^{T} \alpha\left[I^{r}(t)\right]^{\beta} d t \\
=Q-\left[\left(\alpha+\theta Q^{1-\beta}\right) e^{-(1-\beta) \theta T}-\alpha\right]^{1 /(1-\beta)} / \theta^{1 /(1-\beta)} \\
-\frac{\int_{0}^{T} \alpha\left[\left(\alpha+\theta Q^{1-\beta}\right) e^{-(1-\beta) \theta t}-\alpha\right]^{\beta /(1-\beta)} d t}{\theta^{\beta /(1-\beta)}}
\end{gathered}
$$

The profit per unit time for the retailer can be expressed as

$$
\begin{array}{r}
\prod_{1}^{r}=\left(p-c^{r}\right) \int_{0}^{T} \alpha\left[\left(\alpha+\theta Q^{1-\beta}\right) e^{-(1-\beta) \theta t}-\alpha\right]^{\beta /(1-\beta)} d t / T \theta^{\beta /(1-\beta)}-\frac{S}{T} \\
-\frac{h^{r} c^{r} \int_{0}^{T} \alpha\left[\left(\alpha+\theta Q^{1-\beta}\right) e^{-(1-\beta) \theta t}-\alpha\right]^{1 /(1-\beta)} d t / \theta^{1 /(1-\beta)}}{T}-\frac{c^{r} Q^{D, r}}{T}
\end{array}
$$

The profit function (9) is highly nonlinear and cannot be solved by analytical methods. We solve it by using optimization technology on computer and get the following optimal values:

Since the manufacturer is a make -to-order producer, it has a lot-for-lot production policy in response to the retailer's demand. During the production cycle, the change in manufacturer's inventory level is due to the combined effect of production and deterioration: 


$$
\frac{d I^{m}(t)}{d t}=q-\theta I^{m}(t) t_{m} \leq t \leq T
$$

where $t_{m}$ and $T$ are the starting and stopping production times, respectively. With a make-to-order policy, the production quantity of the manufacturer is equal to the demand quantity of the retailer. Therefore, we get:

$$
\int_{0}^{T} I^{r}(t) d t=\int_{t_{m}}^{T} I^{m}(t) d t
$$

Solving the equation (6) by using the method proposed by Spiegel (1960)[18] , we can get:

$$
I^{m}(t)=\frac{q}{\theta}+C_{2} e^{-\theta t},
$$

where $C_{2}$ is constant. By using the boundary condition on inventory $I^{m}\left(t_{m}\right)=0$, we can obtain:

$$
C_{2}=-\frac{q}{\theta} e^{\theta t_{m}}
$$

Therefore, the manufacturer's inventory level at time $t\left(t_{m} \leq t \leq T\right)$ can be expressed as

$$
I^{m}(t)=e^{-\theta\left(t-t_{m}\right)} \int_{t_{m}}^{t} e^{\theta\left(t-t_{m}\right)} q d t=\frac{q}{\theta}-\frac{q e^{-\theta\left(t-t_{m}\right)}}{\theta}
$$

The inventory holding cost in a cycle for the manufacturer is

$$
\begin{aligned}
& H C^{m}=h^{m} c^{m} \int_{t_{m}}^{T} I^{m}(t) d t=h^{m} c^{m} \int_{t_{m}}^{T}\left(\frac{q}{\theta}-\frac{q}{\theta} e^{-\theta\left(t-t_{m}\right)}\right) d t \\
& =h^{m} c^{m} \frac{q}{\theta^{2}}\left[-1+e^{-\theta\left(T-t_{m}\right)}+\left(T-t_{m}\right) \theta\right]
\end{aligned}
$$

The manufacturer's total number of deteriorated goods in a cycle is given by

$$
Q^{D, m}=q\left(T-t_{m}\right)-I^{m}(T)-Q
$$

The profit per unit time for the manufacturer is

$$
\begin{gathered}
\prod_{1}^{m}=\frac{\left(c^{r}-c^{m}\right) Q}{T}-\frac{M}{T}-\frac{H C^{m}}{T}-\frac{c^{m} Q^{D, m}}{T} \\
=\frac{c^{r} Q}{T}-\frac{M}{T}-\frac{h^{m} c^{m} q\left[-1+e^{-\theta\left(T-t_{m}\right)}+\left(T-t_{m}\right) \theta\right]}{T \theta^{2}} \\
-\frac{c^{m} q\left(T-t_{m}\right)}{T}+\frac{c^{m} q}{T \theta}-\frac{c^{m} q e^{-\theta\left(T-t_{m}\right)}}{T \theta}
\end{gathered}
$$

Based on the optimal order quantity and ordering cycle as well as equation (11), we can obtain optimal profit for the manufacturer using (16).

\subsection{The Centralized Supply Chain}

In the centralized supply chain, the order quantity and replenishment cycle are determined by considering the total profit incurred by both the retailer and the manufacturer, so that the overall profit is maximized. The centralized supply chain requires information sharing between manufacture and retailer. Sequential or concurrent engineering will be beneficial to the information sharing.

In this case, the total profit per unit time for the supply chain is the sum of Equation (9) and (16).

$$
\begin{gathered}
\prod_{2}^{s c}=\prod_{1}^{r}+\prod_{1}^{m}= \\
\frac{p \int_{0}^{T} \alpha\left[\left(\alpha+\theta Q^{1-\beta}\right) e^{-(1-\beta) \theta t}-\alpha\right]^{\beta /(1-\beta)} d t}{T \theta^{\beta /(1-\beta)}}-\frac{S}{T} \\
-\frac{h^{r} c^{r} \int_{0}^{T} \alpha\left[\left(\alpha+\theta Q^{1-\beta}\right) e^{-(1-\beta) \theta t}-\alpha\right]^{1 /(1-\beta)} d t}{T \theta^{1 /(1-\beta)}} \\
+\frac{c^{r}\left[\left(\alpha+\theta Q^{1-\beta}\right) e^{-(1-\beta) \theta T}-\alpha\right]^{1 /(1-\beta)}}{T \theta^{1 /(1-\beta)}} \\
-\frac{M}{T}-\frac{h^{m} c^{m} q\left[-1+e^{-\theta\left(T-t_{m}\right)}+\left(T-t_{m}\right) \theta\right]}{T \theta^{2}} \\
-\frac{c^{m} q\left(T-t_{m}\right)}{T}+\frac{c^{m} q}{T \theta}-\frac{c^{m} q e^{-\theta\left(T-t_{m}\right)}}{T \theta}
\end{gathered}
$$

Similarly, since supply chain profit function (17) is highly nonlinear, we solve it by using optimization technology on computer and get the optimal values.

\section{Numerical Study}

To illustrate the effect of the models, we give the following numerical example: $\alpha=0.5$ units per time period, $\beta=0.4, \quad \theta=0.1, \quad S=\$ 10, \quad M=\$ 20, \quad p=\$ 20$ per unit, $q=200$ units, $h^{r}=0.35, h^{m}=0.25, \quad c^{r}=3.5$, $c^{m}=2.0$.

The models were implemented on a personal computer using Mathematica version 5.2. The results are outlined in Table 1 , revealing that the profit for centralized supply chain is greater than that for decentralized supply chain.

Based on the numerical example considered above, we now perform the sensitivity analysis on the effects of changes in the model parameters such as the rate of deterioration, the retailer's purchase cost, the manufacturer's production cost, the retailer's and manufacturer's holding cost on the optimal order quantity for the retailer, the optimal replenishment cycle ( or production cycle ) for the retailer( manufacturer), the optimal supply chain profit, and supply chain profit increase percentages generated by the centralized policy. The effects of changes in the pa rameters values are shown in Table 2 . The sensitivity analysis is performed by changing each of the parameters by $-50 \%,-20 \%,+30 \%$ and $+50 \%$ and keeping the other parameters unchanged. The results are demonstrated in

Table 1. The Sollution Results

\begin{tabular}{|l|l|l|l|l|l|}
\hline Supply chain & $Q^{*}$ & $T^{*}$ & $\Pi^{r}$ & $\Pi^{m}$ & $\Pi^{s c}$ \\
\hline Decentralized & 12.15 & 2.36 & 5.03 & 3.42 & 8.45 \\
\hline Centralized & 20.63 & 2.75 & 4.76 & 4.56 & 9.32 \\
\hline
\end{tabular}


Table 2. Effect of Changes in the Model Parameters

\begin{tabular}{|c|c|c|c|c|c|c|c|c|}
\hline \multirow{3}{*}{ Parameters } & \multirow{3}{*}{ Change (\%) } & \multicolumn{7}{|c|}{ Results of Optimization Procedure } \\
\hline & & \multicolumn{3}{|c|}{ Decentralized Supply Chain } & \multicolumn{3}{|c|}{ Centralized Supply Chain } & \multirow{2}{*}{ Profit Increase (\%) } \\
\hline & & $Q^{*}$ & $T^{*}$ & $\prod_{1}^{S C^{*}}$ & $Q^{*}$ & $T^{*}$ & $\prod_{2}^{S C^{*}}$ & \\
\hline \multirow{4}{*}{$\theta$} & +50 & 10.434 & 2.342 & 6.345 & 18.675 & 2.735 & 7.243 & 14.153 \\
\hline & +20 & 11.876 & 2.351 & 7.421 & 19.538 & 2.747 & 8.256 & 11.252 \\
\hline & -20 & 13.216 & 2.375 & 9.236 & 21.562 & 2.769 & 9.768 & 5.760 \\
\hline & -50 & 13.987 & 2.387 & 10.167 & 24.356 & 2.778 & 10.245 & 0.767 \\
\hline \multirow{4}{*}{$c^{r}$} & +50 & 11.579 & 1.987 & 17.948 & 17.917 & 2.294 & 18.831 & 4.920 \\
\hline & +20 & 11.682 & 2.173 & 14.656 & 19.267 & 2.473 & 15.857 & 8.195 \\
\hline & -20 & 15.876 & 2.563 & 7.063 & 30.013 & 2.931 & 7.771 & 10.024 \\
\hline & -50 & 16.786 & 3.126 & 4.754 & 38.681 & 3.477 & 5.335 & 12.221 \\
\hline \multirow{4}{*}{$c^{m}$} & +50 & 10.342 & 2.031 & 7.346 & 18.625 & 2.334 & 7.658 & 4.247 \\
\hline & +20 & 11.769 & 2.215 & 8.085 & 19.890 & 2.513 & 8.779 & 8.584 \\
\hline & -20 & 16.987 & 2.685 & 11.886 & 31.325 & 2.973 & 13.256 & 11.526 \\
\hline & -50 & 17.769 & 3.264 & 15.538 & 39.706 & 3.517 & 17.543 & 12.904 \\
\hline \multirow{4}{*}{$h^{r}$} & +50 & 10.876 & 2.046 & 7.258 & 17.018 & 2.305 & 7.589 & 4.560 \\
\hline & +20 & 11.986 & 2.263 & 7.982 & 18.845 & 2.433 & 8.648 & 8.344 \\
\hline & -20 & 16.765 & 2.765 & 12.189 & 32.098 & 3.384 & 13.767 & 12.946 \\
\hline & -50 & 17.875 & 3.078 & 15.876 & 40.022 & 3.441 & 18.089 & 13.939 \\
\hline \multirow{4}{*}{$h^{m}$} & +50 & 11.054 & 2.178 & 7.458 & 17.357 & 2.338 & 7.915 & 6.128 \\
\hline & +20 & 12.035 & 2.268 & 8.212 & 18.930 & 2.485 & 8.971 & 9.243 \\
\hline & -20 & 16.497 & 2.589 & 11.687 & 32.021 & 3.264 & 12.893 & 10.319 \\
\hline & -50 & 17.568 & 2.987 & 14.789 & 38.357 & 3.377 & 16.606 & 12.286 \\
\hline
\end{tabular}

table 2. The following observations can be made from

(1) Whether the supply chain is centralized or decentralized, the optimal order quantity, the optimal replenishment cycle (or production cycle), the optimal supply chain profit are decreasing in the deterioration rate. Meanwhile the percentages of supply chain profit increase realized by employing the centralized policy are increasing in the deterioration rates.

(2) Whether the supply chain is centralized or decentralized, the optimal order quantity and replenishment cycle (or production cycle) decrease, while the optimal supply chain profit increases in retailer's unit procurement cost.

(3) Whether the supply chain is centralized or decentralized, the optimal order quantity, the optimal replenishment cycle (or production cycle) and supply chain profit are decreasing in manufacturer's unit manufacturing cost.

(4) Whether the supply chain is centralized or decentralized, the optimal order quantity, the optimal replenishment cycle (or production cycle) and supply chain profit are decreasing in retailer's and manufacturer's inventory holding cost rate.

(5) The percentages of supply chain profit increase realized by employing the centralized policy are increasing in the deterioration rates, but are decreasing in the retailer's purchase cost, the manufacturer's production cost, the retailer's and manufacturer's holding cost.

\section{Conclusions}

This paper has investigated the effect of supply chain coordination for deteriorating goods with stock-dependent demand rate. Two profit models are developed with some assumptions based on exponentially decaying deterioration rates. The numerical study is conducted to demonstrate the effectiveness of the proposed models, and to analyze the impact of supply chain coordination on supply chain profit. Sensitivity analysis is performed to study the impact of different parameters associated with the model, such as the rate of deterioration, the retailer's purchase cost, the manufacturer's production cost, the retailer's and manufacturer's holding cost on the optimal order quantity for the retailer, the optimal replenishment cycle ( or pro- 
duction cycle ) for the retailer( manufacturer), the optimal supply chain profit, and supply chain profit increase percentages generated by the supply chain coordination.

The following observations can be obtained from numerical analysis:

(1) A centralized policy is found to be always superior to a decentralized policy in terms of profit increase, especially when the deterioration rates are high.

(2)Whether the supply chain is centralized or decentralized, the optimal order quantity , the optimal replenishment cycle (or production cycle) , the optimal supply chain profit are decreasing in the deterioration rate, retailer's unit procurement cost, manufacturer's unit manufacturing cost, as well as retailer's and manufacturer's inventory holding cost rate. The optimal supply chain profit are decreasing in the deterioration rate, manufacturer's unit manufacturing cost, as well as retailer's and manufacturer's inventory holding cost rate. Meanwhile it is increasing in retailer's unit procurement cost.

(3)The percentages of supply chain profit increase realized by employing the centralized policy are increasing in the deterioration rates.

The proposed models can be used to analyze gricultural products, fashion goods, drugs and high-tech products supply chain. It is observed that large quantities of these deteriorationg goods displayed in a supermarket tend to generate higher demands. However, the models considered in this paper are somewhat idealized. In reality, when supply chain is coordinated, there are likely to be some costs incurred from information sharing scheme. The information sharing could also affect production, inventory, and other operations. The future research will further consider these factors. These models can be extended in the future research to consider more general deterioration rates. Another extension possibility would be to use other replenishment policies. In addition to profit models, the cost models can also be applied to evaluate the extent of coordination.

\section{Acknowledgement}

This paper is partially supported by Shanghai Shuguang Program under grant no. 06SG48.

\section{REFERENCES}

[1] Chen, J. M., Chen T. H. Effects of joint replenishment and channel coordination for managing multiple deteriorating products in a supply chain, Journal of the operational research society, 56, 2005, pp.1224-1234.

[2] Ghare P. N., Schrader GF. A model for exponentially decaying inventories, Journal of Industry Engineering, 15, 1963, pp.238-243.
[3] Covert R. P., Philip GC. An EOQ model for items with Weibull distribution deterioration, AIIE Transactions, 5, 1973, pp. 323-326.

[4] Philip G. C. A generalized EOQ model for itmes with Weibull distribution deterioration, AIIE Transactions, 6, 1974, pp.159-162.

[5] Tadikamalla, P. R. An EOQ inventory model for items with Gamma distributed deterioration. AIIE Transactions, 10, 1978, pp.78.

[6] Moon I., Lee S. The effects of inflation and time value of money on an economic order quantity model with a random product life cycle, European Journal of Operational Research, 125, 2000, pp.588-601.

[7] Dave, U., Patel L. K. ( $\left.T, S_{i}\right)$ policy inventory model for deterioration items with time proportional demand. Journal of the operational research society, 32, 1981, pp.137-142.

[8] Sachan, R. S.On ( $T, S_{i}$ )policy inventory model deterioration items with time proportional demand. Journal of the operational research society, 35, 1984, pp.1013-1019.

[9] Bahari-Kashani H., Replenishment schedule for deteriorating items with time-proportional demand. Journal of Operational Research Society, 40, 1989, pp.75-81.

[10] Bose, S., Goswami, A., Chaudhuri,K.S., An EOQ model for deteriorating items with linear-dependent demand rate and shortages under inflation and time discounting. Journal of the Operational Research Society, 46, 1995, pp.771-782.

[11] Silver,E. A., Peterson,R., Decision systems for inventory management and production planning, $2^{\text {nd }}$ edition, Wiley, New York, 1985.

[12] Gupta, R., Vrat, P., Inventory model for stock-dependent consumption rate. Opsearch, 23, 1986, pp.19-24.

[13] Mandal, B. N., Phaujdar, S., A note on an inventory model with stock-dependent consumption rate. Opsearch, 26, 1989a, pp.43-46.

[14] Baker, R. C. and Urban,T. L., A deterministic inventory system with an inventory-level-dependent demand rate. Journal of Operational Research Society, 39(9), 1988, pp.823-831.

[15] Datta, T. K., Pal, A. K., Effects of inflation and time-value of money on an inventory model with linear time-dependent demand rate and shortages. European Journal of Operational Research, 52, 1991, pp.1-8.

[16] Mandal, B. N., Phaujdar, S., An inventory model for deteriorating items and stock-dependent consumption rate. Journal of Operational Research Society, 40(5), 1989b., pp.483-488.

[17] Pal, S., Goswami, A., Chaudhuri, K.S., A deterministics inventory model for deteriorating items with stock-dependent demand rate. International Journal of Production Economics, 32, 1993, pp.291-299.

[18] Spiegel, M. R., Applied Differential Equations. Prentice-Hall: Englewood Cliffs, NJ, 1960. 